\title{
Organic Optoelectronic Materials
}

\author{
Van-Chuc Nguyen 10 and Ji-Hoon Lee * $\mathbb{D}$ \\ Future Semiconductor Convergence Technology Research Center, Division of Electronics Engineering, \\ Jeonbuk National University, Jeonju 54896, Korea; chucnguyenvan85@gmail.com \\ * Correspondence: jihoonlee@jbnu.ac.kr
}

Received: 5 November 2020; Accepted: 7 November 2020; Published: 9 November 2020

Organic optoelectronic materials have been widely used in recent electronic devices. The modulation of a refractive index by an external electric or magnetic field can enable the modulation of phase and polarization of the output beam. Various kinds of applications were developed based on this phenomenon: phase and polarization modulation devices, liquid crystal display, holographic devices, etc. With the recent development of flexible electronics and flexible displays, the importance of organic optoelectronic materials has attracted more attention, and reliability in terms of the stretching, bending deformations is required. This Special Issue on "Organic Optoelectronic Materials" covers a broad range research on optoelectronic materials and devices, including a synthesis of materials, optical and electrical properties of materials, fabrication, and instrumentation of optoelectronic devices.

The oxidation of $\mathrm{Cu}$ nanowires (NWs) in ambient conditions is a critical issue that has limited the usage of this material to commercial applications. Recently, Vuong et al. proposed a Nickel coating process on $\mathrm{Cu}$ NWs to overcome this drawback [1]. First, the $\mathrm{Cu}$ NWs $(55.3 \pm 7.1 \mathrm{~nm}$ of diameter, over $100 \mu \mathrm{m}$ of length) were synthesized via a hexadecylamine (HDA)-mediated method, then the pulsed electrode plating technique was used to form the Cu core-Ni shell on the polyethylene terephthalate (PET) film. By effectively controlling the $\mathrm{Cu}-\mathrm{Ni}$ ratio through the pulsed duration, the cupro/nickel NWs-based flexible transparent electrodes were enhanced to be 1000 times more resistant to the oxidation than untreated $\mathrm{Cu}$ NWs. Moreover, the conductivity of the Cu-Ni network was improved after solving the problem of crack development resulting from excessive deposition, and its value was stable after 6000 cycles of bending tests. A flexible polymer-dispersed liquid crystal (PDLC) smart window based on the $\mathrm{Cu}-\mathrm{Ni}$ NWs electrodes was fabricated, and this can prove the possibility of applying the cupro/nickel nanowire in a commercial device.

Luong et al. reported the new synthesis route of a $[\mathrm{O}, \mathrm{N}]$ bidentate $\pi$-expanded ligand system, (E)-1-(n-octylimino)methylpyren-2-ol (2) via seven steps from pyrene [2]. The yield of $70 \%$ can be obtained from the reaction of ligand 2 and [ $\mathrm{PtCl} 2(\mathrm{PhCN}) 2]$ in chlorobenzene with the presence of a base under reflux condition for $2 \mathrm{~h}$. Common spectroscopic and X-ray diffraction results showed that molecular structure of $(\mathbf{2}(\mathbf{P t}))$ consisted of a square planar geometry with a trans-configuration of the ligands. The results from the molecular structure, absorption spectra, electrochemical properties, and phosphorescence characteristics of (2(Pt) ) complex demonstrated that the phosphorescence lifetime of the new isomeric platinum complex is longer than that of the reported with those of the previously reported Pt complex (1(Pt)) containing the isomeric ligands of 2, (E)-2-(n-octylimino)methylpyren-1-ol. In addition, the photophysical properties of pyrene-based materials can be enhanced by the modifications of functional groups on imine group or pyrene rings.

The optical performance degradation in an ion-doped liquid-crystal (LC) cell for light-shutter applications was analyzed by Seo et al. [3]. Electrical circuit modeling was used to analyze the optical and physical characteristics with time and compare them to those of the pure LC cell. The results showed that this model can be used for the analysis of an ion-doped LC cell with high accuracy of impedance. The thickness of the Nernst diffusion layer, electrical double-layer capacitance, haze, mobility, diffusion coefficient in the bright region were decreased, while its surface concentration of 
the ions and resistance were increased with time. Because of the decreases in the current, turbulence, and haze, the ionic molecule amount at the surface electrode increased, however, that amount in the bulk region decreased. The measured results demonstrated that the performance degradation of ion-doped LC cell was mainly attributed to the ion accumulation at the surfaces of the electrodes.

The three-dimensional (3D) crosstalk level of multi-view 3D displays was improved by Lee et al. using a lens array with small f-number for wide 3D viewing window application [4]. They reported that a polarization-dependent switching liquid crystal (LC)-based gradient refractive index (GRIN) lens array could be switched between $2 \mathrm{D}$ and $3 \mathrm{D}$ viewing modes. The transverse spherical aberration (TSA) properties of lens arrays and their effects on the 3D crosstalk levels can be investigated by changing the interfacial curvature profiles. For the GRIN lens with a f-number of 1.08, the TSA properties were considerably improved when the high-order polynomial aspherical curvature profile was used between the plano-concave isotropic polymer layer and the plano-convex birefringent LC layer. The average adjacent crosstalk levels within the entire viewing window $\left(50^{\circ}\right)$ were $73.3 \%, 68.5 \%$, and $60.0 \%$ for the quadratic polynomial aspherical, spherical, and high-order polynomial aspherical curvatures, respectively, in the designed multi-view (43-view) 2D/3D switchable mobile displays containing the polarization-dependent-switching LC GRIN lens arrays with the three types of interfacial curvatures.

The small luminescence dissymmetry factor (glum) in a nano-segregated phase system, generated by the phase separation between helical nanofilaments (HNFs; originating from an achiral bent-core molecule) and a liquid-crystalline (LC) smectic A (SmA) phase (originating from an achiral rod-like mesogen), was enhanced by Lee et al. [5]. The glum value in the nano-segregated phase between the HNFs and LC SmA phase was larger than that between the HNFs and LC nematic phase. The enhancement of the glum was due to the larger order parameter (S) of the dye molecules in the $\mathrm{SmA}$ phase compared to that in the nematic phase. They concluded that the glum value was strongly influenced by the $S$ value of the fluorescent dye molecules, which was doped into the embedded LC phase between the HNFs.

The present Special Issue on "Organic Optoelectronic Materials" can be considered as a report reviewing the recent researches on the synthesis, analysis, and application optoelectronic materials and devices.

Conflicts of Interest: The authors declare no conflict of interest.

\section{References}

1. Vuong, D.-T.; Hoang, H.-M.; Tran, N.-H.; Kim, H.-C. Pulsed Electrodeposition for copper nanowires. Crystals 2020, 10, 218. [CrossRef]

2. Luong, X.-D.; Nguyen, X.-T. Metal complexes of $\pi$-Expanded Ligand (7): Syntheses, structures and properties of Pt(II) complexes containing the Isomeric 1- and 2-Alkyliminomethyl Pyrene ligands. Crystals 2020, 10, 476. [CrossRef]

3. Seo, J.-H.; Huh, J.-W.; Sohn, H.-J.; Lim, E.; Yoon, T.-H. Analysis of optical performance degradation in an ion-doped liquid-crystal cell with electrical circuit modeling. Crystals 2020, 10, 55. [CrossRef]

4. Lee, T.-H.; Joo, K.-I.; Kim, H.-R. Switchable lens design for multi-view 2D/3D switching display with wide-viewing window. Crystals 2020, 10, 418. [CrossRef]

5. Lee, J.-J.; Choi, S.-W. Enhancement of luminescence dissymmetry factor in nano-segregated phase generated by phase separation between helical Nanofilaments and liquid-crystalline Smectic a phase. Crystals 2020, 10, 952. [CrossRef]

Publisher's Note: MDPI stays neutral with regard to jurisdictional claims in published maps and institutional affiliations. 